\title{
stabilité d'un ensemble de matériaux sous contraintes
}

\author{
par \\ R. Poirot \\ Ingénieur au CERCHAR
}

STABILITE D'UN ENSEMBLE DE MATERIAUX SOUS CONTRAINTES

L'étude des énergies mises en jeu au cours d'un essai de compression simple permet d'interpréter le comportement explosif de certains échantillons. Elle résulte d'une instabilité mécanique de l'ensemble constitué par la presse et l'échantillon.

Ce résultat est ensuite généralisé pour expliquer le comportement d'un échantillon hétérogène au laboratoire et d'un ensemble de terrains à proximité d'une excavation profonde.

\section{BEHAVIOUR OF COMPOSITE MEDIUM UNDER COMPRESSION}

Energy analysis during uniaxial compression test explains the explosion which occurs with some types of rocks. It is due to the instability of the combination of the sample and the testing machine.

A generalisation of this analysis explains the behaviour of an heterogeneous rock sample compression test, or that of a stratified rock mass around an excavation. 
Comité Français de Mécanique des Roches 


\title{
stabilité d'un ensemble de matériaux sous contraintes
}

\author{
par R. POIROT
}

Dans le rapport général du thème 4: Mécanique des Roches appliquée aux excavations souterraines - Denver 1974 - pages 1106 et suivantes, le Pr. M.D.G. SAlamon esquisse une méthode d'analyse du comportement d'un échantillon au cours d'un essai de compression simple. En partant des mêmes hypothèses, il est possible d'étudier le problème dans sa généralité et de proposer quelques extensions pratiques à cette analyse.

\section{MECANISME D'UN ESSAI DE COMPRESSION SIMPLE}

L'échantillon est placé entre les plateaux de la presse et on suit sa déformation $s_{1}$ mesurée par un comparateur en fonction de l'effort exercé P. Sa loi de comportement est donnée par la relation :

$$
\mathrm{P}=f\left(s_{1}\right)
$$

La presse s'allonge élastiquement en fonction de l'effort exercé suivant une loi linéaire :

$$
\mathrm{Q}=k s_{2}
$$

$k$ étant la raideur de la presse.

La figure $1 \mathrm{~b}$ montre comment on passe du schéma de la presse à un schéma équivalent, plus commode pour interpréter les phénomènes mis en jeu.

En supposant l'avance des plateaux suffisamment lente, on a équilibre entre la force de serrage exercée par la presse et la résistance de l'échantillon,

$$
\mathrm{P}=\mathrm{Q}
$$

ou $f\left(s_{1}\right)=k s_{2}$

et en désignant par $\gamma$ l'avance du plateau mobile,

$$
\gamma=s_{1}+s_{2}
$$

Avant d'atteindre la rupture, toute augmentation du serrage provoque une augmentation de $\gamma$ qui se répartit suivant $s_{1}$ et $s_{2}$ en conservant l'égalité (4).

La figure $1 \mathrm{c}$ donne une représentation géométrique des équations (1) et (4) qui est condensée dans la figure $1 \mathrm{~d}$. Les aires des triangles $\mathrm{OAB}$ et $\mathrm{OA}^{\prime} \mathrm{B}^{\prime}$ représentent les énergies fournies à l'échantillon et à la presse. Il est important de noter que le schéma équivalent décrivant le système ne comporte pas la source d'énergie de serrage - pompe hydraulique par exemple.

La représentation géométrique permet de calculer facilement l'énergie fournie au système au cours d'un essai de compression :

$$
\begin{array}{rlrl} 
& \text { l'échantillon a reçu } \mathrm{W}_{\mathrm{E}} & =\int_{0}^{s_{\mathrm{i}}} f(s) d_{s} \\
& \text { la presse : } & \mathrm{W}_{\mathrm{P}} & =\int_{s_{\mathrm{l}}}^{\gamma} k(\gamma-s) d s
\end{array}
$$

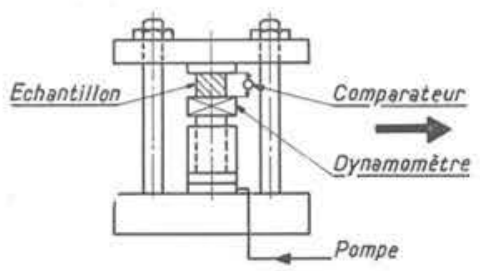

Fig. 1a-SCHEMA D'UNE PRESSE
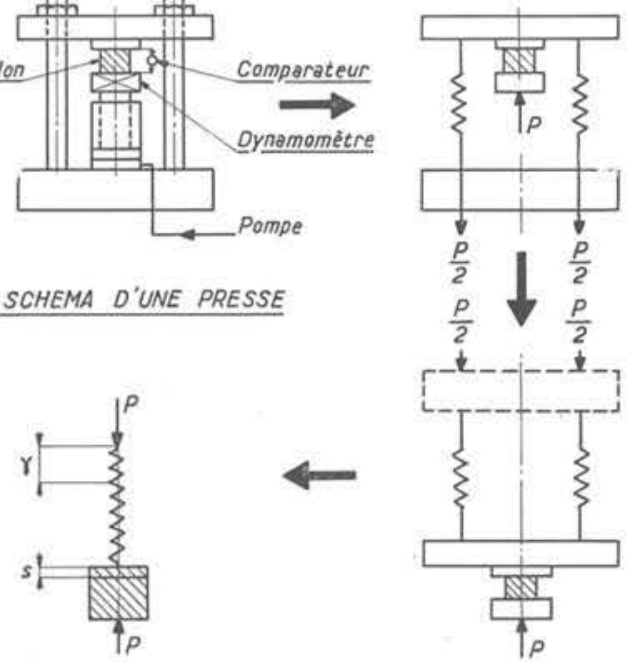

Fig. 10. SCHEMA EQUIVALENT
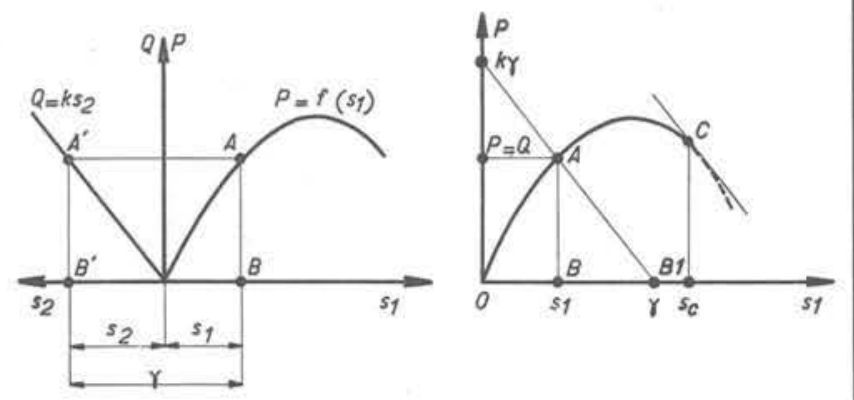

Fig.1C_DIAGRAMME DE FONCTIONNEMENT

Fig.1d_DIAGRAMME CONOE,...

Fig. 1. - Essai de compression simple. 
$\mathrm{W}_{\mathrm{P}}$ est représenté par l'aire du triangle $\mathrm{OA}^{\prime} \mathrm{B}^{\prime}$. soit

$$
\mathrm{W}_{\mathrm{P}}=\frac{\left[f\left(s_{1}\right)\right]^{2}}{2 k}
$$

L'énergie totale reçue est :

$$
\mathrm{W}_{\mathrm{R}}=\int_{0}^{s_{1}} f(s) d s+\frac{\left[f\left(s_{1}\right)\right]^{2}}{2 k}
$$

Cette énergie passe par un maximum si :

$$
\frac{d \mathrm{~W}_{\mathrm{R}}}{d s_{1}}=0
$$

ou $k+f^{\prime}\left(s_{1}\right)=0$

Cette circonstance se produit si la courbe représentative de $\mathrm{P}=f\left(s_{1}\right)$ admet une tangente de pente $-k$. A ce moment, l'équilibre est rompu car la presse libère spontanément plus d'énergie que l'échantillon ne peut en absorber.

\section{GENERALISATION}

\subsection{Compression de deux matériaux de compressibilités différentes}

Le schéma équivalent et sa représentation géométrique peuvent être adaptés en remplaçant la caractéristique linéaire par la loi de comportement du deuxième matériau.

$$
\mathrm{Q}=g\left(s_{2}\right)
$$

Le calcul étant plus compliqué que dans le cas précédent, nous le donnerons en annexe et nous utilisons la représentation géométrique (fig. 2).
Le système constitué par les deux échantillons (P) et (Q) sera instable pour une déformation critique $s_{c}$ du moins résistant, si par translation parallèle à l'axe $\mathrm{O} s_{1}$, les deux courbes représentatives des lois de comportement $\mathrm{P}=f\left(s_{1}\right)$ et $\mathrm{Q}=g\left(s_{2}\right)$ admettent une tangente commune.

\subsection{Compression de plusieurs matériaux}

On commencera par les classer en fonction de leur résistance à la rupture, puis on examinera le comportement du moins résistant vis-à-vis de ceux qui sont à son contact.

Fig. 2. - Compression de deux échantillons de matériaux différents.

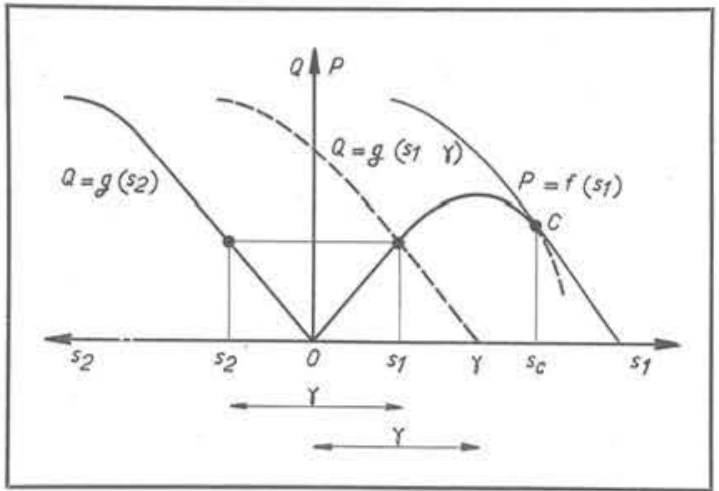

\section{CONSEQUENCES PRATIQUES}

3.1. Nous n'avons pas fait appel à la notion de contrainte et avons utilisé des diagrammes (force, écrasement) et non (contrainte, déformation relative).

Ceci nous a permis la représentation directe des énergies mises en jeu et d'établir la condition de stabilité. II en résulte immédiatement un effet de la taille de l'échantillon sur le résultat de l'essai de compression au-delà de la rupture.

En supposant des échantillons homogènes et pour une presse de raideur $k$, si on passe d'un échantillon à son homothétique (fig. $3 \mathrm{a}$ ), de rapport $\alpha$, pour un même état de tension :

- les efforts sont multipliés par $\alpha^{2}$,

- les déformations par $\alpha$.

Vis-à-vis de l'échantillon initial, tout se passe comme si la presse avait une raideur divisée par $\alpha$.

Par conséquent, une même presse sera d'autant plus raide que les échantillons seront plus petits.
3.2. En réalité, l'échantillon n'est jamais homogène et de plus les contacts entre les plateaux de la presse et les faces de l'échantillon induisent une perturbation dans le champ des contraintes de telle sorte que la loi de comportement $\mathrm{P}=f\left(s_{1}\right)$ est une loi statistique.

Chaque volume élémentaire $\mathrm{V}_{i}$ a une loi $\mathrm{P}_{i}=f\left(s_{1}\right)$ et on peut imaginer que deux éléments contigus donnent lieu à un petit groupement instable au-delà d'une certaine déformation. Ceci expliquerait les dents de scie effectivement observées sur l'enregistrement effort-déformation d'un essai et qui ont une pente correspondant à la raideur de la presse.

3.3. La raideur de la presse est une caractéristique purement mécanique et il est abusif de dire qu'un asservissement destiné à imposer une loi de déformation rend la presse « raide ».

Jusqu'au seuil d'instabilité nous n'avons pas eu à faire intervenir le facteur temps. Pourtant, il intervient 


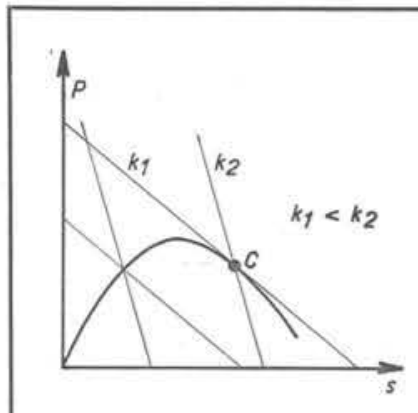

EFFET RAIDEUR DE LA PRESSE

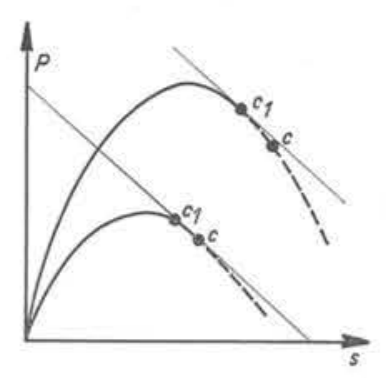

EFFET D'ECHELLE

\section{Fig. $3 a_{-}$ESSAI DE COMPRESSION SIMPLE}

Fig. 3. - Applications.
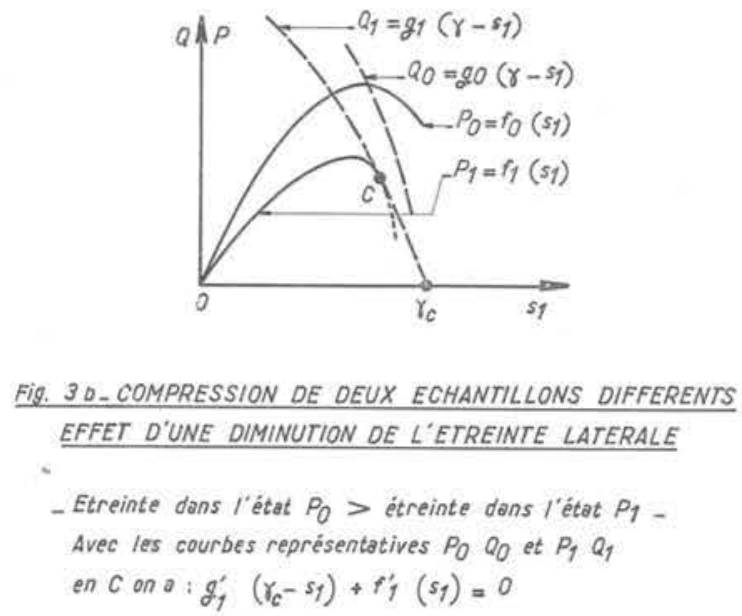

Fig. 30-COMPRESSION DE DEUX ECHANTILLONS DIFFERENTS EFFET D'UNE DIMINUTION DE L'ETREINTE LATERALE - Etreinte dans l'état $P_{0}>$ étreinte dans l'état $P_{1}$ Avec les courbes représentatives $P_{0} Q_{0}$ et $P_{1} Q_{1}$ en $c$ on a: $g_{1}^{\prime}\left(\gamma_{c}-s_{1}\right)+f_{1}^{\prime}\left(s_{1}\right)=0$

déjà, car on observe que le matériau semble d'autant plus raide que la vitesse d'application de l'effort est grande. Ceci laisse supposer qu'il n'y a pas simultanéité entre la cause - effort - et l'effet - fissuration; en d'autres termes, la propagation des fissures ne serait pas très rapide. L'étude du bruit - microséismicité - pendant un essai de compression est de nature à confirmer ce point de vue.

Au-delà du seuil d'instabilité, pour éviter la rupture explosive, il faut que la presse restitue à l'extérieur l'énergie que l'échantillon n'est pas capable d'absorber; l'expérience montre que c'est possible avec des asservissements dont le temps de réponse est de $1 / 10$ à $1 / 100$ de seconde et d'autant plus facile à réaliser que la presse est raide, puisque l'énergie qu'elle a emmagasinée est inversement proportionnelle à sa raideur. Le succès des essais avec les presses asservies confirme lui aussi l'hypothèse de la relative lenteur du développement des fissures.
3.4. Cette analyse de la stabilité permet, à partir d'une étude au laboratoire, de prévoir des conditions favorables à certains coups de terrains, qui pourraient survenir dans le creusement d'un tunnel ou dans une exploitation minière.

En effet, un terrain peut être broyé sous triple étreinte; si l'expansion est rendu possible par une excavation (galerie ou taille) le régime des tensions est modifié et la condition d'instabilité peut être remplie (fig. 3 b).

Un autre exemple est fourni par une veine de charbon contenant un banc rocheux. Si le charbon, plus déformable que la roche, a localement une résistance supérieure à cette dernière, on constate des explosions de la veine : le banc rocheux vole en éclats.

On peut confirmer ce résultat par un essai de laboratoire qui consiste à comprimer un échantillon rocheux entre deux plaques de caoutchouc. $\mathrm{Si}$ on atteint la rupture, elle est toujours violente.

\section{CONCLUSIONS}

L'analyse du comportement d'un ou plusieurs échantillons au cours d'un essai de compression permet d'interpréter les phénomènes qui se passent au-delà de la limite de rupture conventionnelle et met en lumière l'interaction entre la presse et l'échantillon.
Le comportement explosif de certains matériaux est lié à leur perte de résistance en fonction de l'écrasement.

La prévision du mécanisme de rupture in situ nécessite la connaissance des courbes effort-déformation 
au-delà de la rupture, pour chacun des terrains en présence. Or, la détermination de ces courbes peut être fortement perturbée par la presse d'essai.

Si l'essai à la presse a un caractère explosif, il faut ou choisir une presse plus raide éventuellement asservie, ou diminuer la taille de l'échantillon dans la mesure où la struture du matériau permet une réduction d'échelle.

Les coups de terrains dont l'origine est liée au mécanisme proposé pourraient être d'une part prévus et évités ou minimisés dans leurs effets en agissant sur les caractéristiques des matériaux - par exemple en augmentant le module de déformation naturel par frettage ou boulonnage.

L'interprétation des phénomènes observés pendant l'écrasement du matériau met en évidence la relative lenteur de la propagation des fissures qui semblent progresser par sauts au cours du broyage de volumes de petites dimensions.

Nous pensons que le mode de propagation des fissures est un des éléments déterminant de la pente de la courbe effort-déformation au-delà de la limite de rupture conventionnelle et envisageons de l'étudier au laboratoire et sur le terrain à proximité d'excavations profondes. 


\section{DETERMINATION ANALYTIQUE DE LA STABILITE DANS LE CAS DE PLUSIEURS MATERIAUX}

Comme cela a été exposé au paragraphe 2, il suffit de faire cette détermination pour deux matériaux quelconque $(\mathrm{P})$ et $(\mathrm{Q})$ ayant des courbes caractéristiques

$$
\begin{aligned}
& \mathrm{P}\left(s_{1}\right)=f\left(s_{1}\right) \\
& \mathrm{Q}\left(s_{2}\right)=g\left(s_{2}\right)
\end{aligned}
$$

avec $\gamma=s_{1}+s_{2}$.

A l'équilibre, on a :

$$
f\left(s_{1}\right)=g\left(s_{2}\right)
$$

ou

$$
f\left(s_{1}\right)=g\left(\gamma-s_{1}\right) \quad\left(\gamma \text { est une fonction de } s_{1}\right)
$$

L'énergie fournie au système, constitué par les deux matériaux, au cours d'une déformation $s_{1}$ de (P) est :

$$
\begin{aligned}
& \mathrm{W}_{\mathrm{R}}=\int_{0}^{s_{1}} f\left(s_{1}\right) d s_{1}+\int_{0}^{s_{2}} g\left(s_{2}\right) d s_{2} \\
& \text { Posons } \mathrm{G}\left(s_{2}\right)=\int_{0}^{s_{2}} g\left(s_{2}\right) d s_{2} \\
& \frac{d \mathrm{G}\left(s_{2}\right)}{d s_{1}}=\frac{d \mathrm{G}\left(s_{2}\right)}{d s_{2}} \frac{d s_{2}}{d s_{1}} \\
& =g\left(\gamma-s_{1}\right)\left(\frac{d \gamma}{d s_{1}}-1\right)
\end{aligned}
$$

En dérivant $f\left(s_{1}\right)=g\left(\gamma-s_{1}\right)$

$$
\begin{gathered}
f^{\prime}\left(s_{1}\right)=g^{\prime}\left(\gamma-s_{1}\right)\left(\frac{d \gamma}{d s_{1}}-1\right) \\
\frac{d \gamma}{d s_{1}}-1=\frac{f^{\prime}\left(s_{1}\right)}{g^{\prime}(\gamma-1)} \\
\frac{d \mathrm{G}\left(s_{2}\right)}{d s_{1}}=\frac{g\left(\gamma-s_{1}\right)}{g^{\prime}\left(\gamma-s_{1}\right)} \cdot f^{\prime}\left(s_{1}\right) \\
\frac{d \mathrm{~W}_{\mathrm{R}}}{d s_{1}}=f\left(s_{1}\right)+\frac{g\left(\gamma-s_{1}\right)}{g^{\prime}\left(\gamma-s_{1}\right)} \cdot f^{\prime}\left(s_{1}\right) \\
\text { comme } \quad f\left(s_{1}\right)=g\left(\gamma-s_{1}\right) \\
\frac{d \mathrm{~W}_{\mathrm{R}}}{d s_{1}}=\frac{f\left(s_{1}\right)}{g^{\prime}\left(\gamma-s_{1}\right)}\left(g^{\prime}\left(\gamma-s_{1}\right)+f^{\prime}\left(s_{1}\right)\right) \\
\text { Si } g^{\prime}\left(\gamma-s_{1}\right)+f^{\prime}\left(s_{1}\right)=0 \\
\frac{d \mathrm{~W}_{\mathrm{R}}}{d s_{1}} \text { s'annule en changeant de signe. }
\end{gathered}
$$

Le système est instable, sauf si des deux courbes représentatives $\mathrm{P}=f\left(s_{1}\right)$ et $\mathrm{Q}=g\left(\gamma-s_{1}\right)$ sont osculatrices, mais compte tenu des dispersions des caractéristiques des matériaux, on peut pratiquement écarter ce cas. 
